\title{
Uso de periódicos eletrônicos nas instituições do ensino superior e de pesquisa em Moçambique
}

\author{
Use of electronic journals in institutions of higher education and research centers in \\ Mozambique
}

\begin{abstract}
Ranito Zambo Waete
Mestre em Ciência da Informação pela Universidade Federal de Minais Gerais - UFMG. Assistente Universitário da Escola de Comunicação e Artes da Universidade Eduardo Mondlane, Moçambique.

E-mail: rwaete@yahoo.com.br

Maria Aparecida Moura

Doutora em Comunicação e Semiótica pela Pontifícia Universidade Católica de São Paulo Professora Titular da Escola de Ciência da Informação da Universidade Federal de Minais Gerais - UFMG.

E-mail: mamoura@eci.ufmg.br

Manuel Valente Mangue

Doutor em Ciência da Informação pela Universidade Federal de Minais Gerais - UFMG.

Professor Auxiliar da Escola de Comunicação e Artes da Universidade Eduardo Mondlane, Moçambique.

E-mail: mvmangue@yahoo.com.br
\end{abstract}

\section{Resumo}

O estudo realizado tomou como referência a centralidade e as repercussões dos Portais de Períodicos Eletrônicos disponíveis em Moçambique para a pesquisa científica. Foram analisados os graus de aceitação e utilização de Periódicos Eletrônicos e dos Portais de Periódicos do INASP, HINARI, AGORA e CAPES por professores e pesquisadores de Instituições do Ensino Superior e de Pesquisa em Moçambique. A metodologia contou com a combinação de abordagens qualitativas e quantitativas. Também serviu-se da pesquisa documental, para tanto, utilizou-se dos documentos oficiais e técnicos coletados junto aos órgãos do Governo de Moçambique, voltados para políticas públicas de informação, ciência e tecnologia. Os resultados obtidos permitiram concluir que tanto os professores quanto pesquisadores utilizam de forma rotineira periódicos eletrônicos em suas atividades de ensino e pesquisa. Os mesmos resultados indicaram que a minoria dos professores e pesquisadores desconhece a existência desses recursos eletrônicos e apontarem a barreira linguística como um grande entrave para o acesso e utilização dos períodicos eletrônicos disponiveis nos Portais.

Palavras-chave: Periódicos Científicos Eletrônicos; Comunicação Científica; Portais de Periódicos.

\begin{abstract}
The study took as reference the centrality and the impact of the Portals Electronics in Mozambique available for scientific research in the country. Therefore, we analyzed the degree of acceptance and use of Electronic Journals and Journals Portals of INASP, HINARI, AGORA and CAPES for teachers and researchers in Mozambique. The methodology included a combination of qualitative and quantitative approaches. Also served up the documentary research, therefore, it was used in official documents and technicians collected from the bodies of the Government of the Mozambique, facing public policy information, science and technology. The results suggest that both teachers and researchers from routinely use electronic journals in their teaching and research activities. The same results showed that minority teachers and researchers, they are unaware of the existence of electronic journals and Portals Journals. Regarding the degree of satisfaction with the use of portals, they are reasonably satisfied, although they see language as a major barrier for accessing and using electronic journals available in Portals.
\end{abstract}

Keywords: Electronic Scientific Journals, Scientific Communication; Portals Journals.

InCID: R. Ci. Inf. e Doc., Ribeirão Preto, v. 4, n. 2, Ed. esp., p. 165-184, jul./dez. 2013. 


\section{Introdução}

O presente estudo de caráter exploratório, utiliza de revisão bibliográfica e documental pertinente para a fundamentação e o tratamento analítico do corpus do estudo. A bibliografia estudada circunscreve-se principalmente nos campos da Biblioteconomia, Ciências da Informação e da Computação. Os textos selecionados são trabalhos científicos, como artigos de periódicos, teses, dissertações e livros, dos tópicos relacionados à comunicação científica, periódicos científicos eletrônicos, repositórios institucionais e acesso aberto (open access) cujas temáticas incidem, direta ou indiretamente, sobre o tema proposto, norteando os elementos do referencial teórico desenvolvido.

O uso de periódicos eletrônicos, embora esteja em franca expansão em Moçambique, o seu impacto tem sido pouco estudado, sobretudo nos aspectos que dizem respeito à sua aceitação e efetivo uso pela comunidade científica. O nosso propósito nesse estudo é buscar preencher tal lacuna. A consciência dos desafios e das oportunidades do uso e acesso a esses recursos tem vindo a ganhar ímpeto e peso na comunidade científica moçambicana. Assim, no âmbito da agenda nacional de "repensar o ensino superior e a pesquisa", o Governo de Moçambique acredita que a disponibilização gratuita dos recursos eletrônicos, entre eles, os periódicos eletrônicos, Portais de Periódicos, e-book, entre outros, aos alunos, professores e pesquisadores das Instituições de Ensino Superior e de Pesquisa (IESP), pode constituir um grande potencial para impulsionar a pesquisa científica e tecnológica no país. É nesses termos que, para o presente estudo, levantam-se as seguintes questões: (1) qual é a percepção sobre o acesso e uso dos periódicos eletrônicos por professores e pesquisadores das IESP em Moçambique e; (2) quais fatores que intervêm no grau de adesão dos periódicos eletrônicos por professores e pesquisadores das IESP? Isso implica a análise da infraestrutura físico-tecnológica, bem como a necessidade de se fazer um mapeamento dos recursos acessados e utilizados pelos usuários como forma de identificar as suas percepções e necessidades.

No sentido de estudar os mecanismos de acesso e uso de Portais de Periódicos eletrônicos, o estudo teve como objetivo geral, analisar o grau de aceitação e utilização dos Portais de Periódicos do INASP ${ }^{1}$, HINARI $^{2}$, AGORA $^{3}$ e CAPES $^{4}$ por professores e pesquisadores de IESP

\footnotetext{
${ }^{1}$ INASP - International Network for the Availability of Scientific Publications

InCID: R. Ci. Inf. e Doc., Ribeirão Preto, v. 4, n. 2, Ed. esp., p. 165-184, jul./dez. 2013.
} 
moçambicanas. Para tanto, foram definidos os seguintes objetivos específicos: (1) identificar a adesão e o uso de Portais de Periódicos por professores e pesquisadores das IESP e mapear o contexto institucional em que ocorrem as suas atividades de docência e pesquisa; (2) identificar e sistematizar as práticas do uso dos Portais na percepção e recepção dos professores e pesquisadores de IESP; (3) iidentificar as fontes de informação (impressas ou eletrônicas) preferencialmente consultadas por professores e pesquisadores de IESP; (4) identificar fatores que podem explicar o uso ou não-uso dos Portais; (5) verificar e explicar, a partir da análise documental, as ações governamentais na implementação das políticas públicas de acesso a informação.

O estudo realizado teve também como perspectiva levantar elementos que permitam discutir e apoiar as ações governamentais na implementação das políticas públicas de acesso à informação em ciência e tecnologia. Em linhas gerais, espera-se que os resultados deste estudo subsidiem o planejamento e o uso efetivo dos Portais de Periódicos de INASP, HINARI, AGORA e CAPES com vista a contribuir para o desenvolvimento do país, particularmente, no ensino superior e pesquisa.

\section{Fundamentação Teórica}

Em que pese a existência de uma variedade de publicações eletrônicas, este estudo centrase na análise dos periódicos eletrônicos, considerados por Cruz et al. (2003), como uma das formas mais rápidas de divulgação dos resultados de pesquisas pela comunidade científica. No entanto, definir periódico eletrônico dentro da complexa conceituação de publicação eletrônica não é tarefa aparentemente fácil. Assim, das diversas definições apresentadas por diferentes autores, utilizaremos o conceito que define o periódico eletrônico tanto da publicação apenas disponibilizada em meio eletrônico como aquela que contém versões em ambos os suportes. $\mathrm{O}$ nosso argumento é que, para o nosso usuário (professores e pesquisadores de IESP em Moçambique), a distinção entre a existência do título apenas em formato eletrônico e àqueles que possuem também a versão impressa não é primordial.

\footnotetext{
${ }^{2}$ HINARI - Access to Research in Health Programme

${ }^{3}$ AGORA - Access to Global Online Research in Agriculture

${ }^{4}$ CAPES - Coordenação de Aperfeiçoamento de Pessoal de Nível Superior

InCID: R. Ci. Inf. e Doc., Ribeirão Preto, v. 4, n. 2, Ed. esp., p. 165-184, jul./dez. 2013.
} 
Assim, o alinhamento do nosso objeto de estudo apontou para três focos: (a) foco conceitual: publicação eletrônica é um spectrum amplo de possibilidades conceituais que vai desde o documento produzido através de processadores de texto até o documento produzido e disponibilizado unicamente via rede eletrônica. O papel da publicação eletrônica no âmbito da comunicação formal da ciência, e como esta se relaciona com periódicos eletrônicos (CASTEDO, 2009; LANCASTER, 1995; OLIVEIRA, 2008; CRUZ et al., 2003; ARAÚJO et al., 2006; MEADOWS, 2001); (b) foco no acesso e uso dos periódicos eletrônicos: utilização dos periódicos eletrônicos nas IESP e benefícios e preocupações que estes periódicos trouxeram aos bibliotecários, procurando compreender o nível de conhecimento que a comunidade acadêmica tem relativamente à existência e disponibilidade destes recursos, conhecer as práticas e frequências do seu acesso e uso, aferir vantagens e desvantagens e perceber as principais consequências (COSTA, 2008; OLIVEIRA, 2006; CENDÓN; RIBEIRO, 2008; CENDÓN et al., 2009; CUNHA; CENDÓN, 2010); (c) foco no acesso e uso dos portais de periódicos: o acesso aos Portais de Periódicos de INASP, HINARI, ÁGORA e CAPES, que oferecem acesso livre (open access) aos periódicos nas IESP (CENDÓN; RIBEIRO, 2008; CENDÓN et al., 2009; CUNHA; CENDÓN, 2010; MAIA, 2005; PEEK; POMERANTZ, 1998; DIAS, 2001; ROWLANDS, 2007).

O estudo procurou compreender o nível de conhecimento que a comunidade científica tem relativamente à existência e disponibilidade destes recursos eletrônicos, conhecer práticas e frequência do seu uso, aferir vantagens e desvantagens e perceber as principais consequências, benefícios e preocupações que estes recursos trouxeram também aos técnicos de biblioteca (OLIVEIRA, 2006; COSTA, 2008). Finalmente, Cendón; Ribeiro (2008); Cendón at al. (2009); Cunha; Cendón (2010); Maia (2005), estudaram o acesso aberto ao Portal de Periódicos da CAPES, que oferece acesso a artigos de periódicos eletrônicos em instituições de ensino superior. Estes estudos facilitaram a abordagem comparativa entre o nosso objeto de estudo em Moçambique com a abordagem brasileira, em termos de disponibilização de periódicos eletrônicos via Portais.

Há poucos estudos sistemáticos sobre o uso dos Portais de Periódicos de INASP, HINARI, AGORA e CAPES e dos periódicos eletrônicos pelas Instituições de Ensino Superior e de Pesquisa (IESP) em Moçambique. Todavia, a maior parte dos estudos emana de pesquisas que 
analisam a aplicação das tecnologias de informação e comunicação em diferentes ambientes (MANGUE, 2007; NHARRELUGA, 2006; ZIMBA, 2010; MONTEIRO, 2010, entre outros).

\subsection{Gênese das Instituições de Ensino Superior e de Pesquisa em Moçambique}

Falar sobre a gênese do Subsistema de Educação Superior em Moçambique é fazer uma abordagem mais ou menos histórica, isto é, a partir da sequência de eventos que o criam. Desta forma, podemos dividir em dois grandes momentos o Subsistema do Ensino Superior (SES) em Moçambique, tendo a Lei $n^{o} 1 / 93$, de 24 de Junho, como grande marco histórico divisor. O primeiro marco importante, definido pelo Decreto 44.530, de 21 de Agosto de 1962, foram criados os Estudos Gerais Universitários de Moçambique, como resposta às críticas dos movimentos nacionalistas das colônias portuguesas, acusando-a de nada fazer pelo desenvolvimento dos povos das colônias. Pelo Decreto-Lei 43.799, de Dezembro de 1968, foi criada a Universidade de Lourenço Marques (ULM). Como resultado das profundas transformações político-sociais decorrentes da ascensão do país à independência, em 1975, as mudanças políticas vividas neste período tiveram repercussões sobre o ensino superior no país. Assim, em 1976, a ULM passou a chamar-se de Universidade Eduardo Mondlane (UEM), em homenagem ao $1^{\circ}$ Presidente da FRELIMO ${ }^{5}$.

Segundo marco histórico importante é a Lei $n^{o} 1 / 93$, de 24 de Junho (Lei do Ensino Superior), que regula atividades de ensino superior no país. Este instrumento cria o quadro legal para a execução das atividades do ensino superior em Moçambique e permite a intervenção do setor privado na educação superior. Hoje, são no total 26 instituições privadas de ensino superior. Ao todo, entre públicas e privadas, são 44 instituições, entre universidades, institutos superiores e politécnicos, escolas superiores e academias.

À semelhança do Subsistema de Educação Superior (SES), quando se aborda o papel de Sistema Nacional de Pesquisa (SNP) em Moçambique, deve-se ter em conta os diferentes momentos históricos a que o país esteve sujeito. Assim, havia no país uma única instituição, o Instituto de Investigação Cientifica de Moçambique (IICM), considerado a "catedral" das

\footnotetext{
${ }^{5}$ FRELIMO é o acrónimo da Frente de Libertação de Moçambique, uma força política oficialmente fundada em 1962, com o objectivo de lutar pela independência de Moçambique do domínio colonial português.

InCID: R. Ci. Inf. e Doc., Ribeirão Preto, v. 4, n. 2, Ed. esp., p. 165-184, jul./dez. 2013.
} 
ciências sociais coloniais, pois era neste Instituto que se realizava toda a pesquisa científica. $\mathrm{O}$ IICM foi fundado em 1955, e era controlado a partir de Lisboa pela Junta de Investigações do Ultramar. O principal objetivo do Instituto era a pesquisa científica, tecnológica, econômica e sociológica. O seu campo de pesquisa compreendia os ramos das ciências biológicas, ciências da terra e as ciências sociais, sendo que, na década de 1950, era o único organismo em Moçambique. O IICM, nos anos subsequentes à independência nacional, em 1976, o Instituto seria então integrado, por decreto do então Ministério da Educação e Cultura, na estrutura administrativa universitária.

No período pós-independência, os professores universitários e pesquisadores portugueses abandonaram o país em grande número, pondo a risco a continuidade das atividades de ensino superior e de pesquisa. Desta forma, falar do ensino superior é falar da pesquisa porque, segundo o discurso político, ambos tem como principais objetivos contribuir para o desenvolvimento do país e a erradicação da pobreza absoluta.

\subsection{Comunicação Científica e Periódicos Científicos}

A comunicação científica é entendida, no âmbito deste artigo, segundo GARVEY ${ }^{6}$ (1979 apud DIAS, 2009), como a "troca de informações entre membros da comunidade científica, incluindo, atividades associadas à produção, disseminação e uso da informação". Porém, a comunicação científica está vinculada ao funcionamento da comunidade científica, compreendendo a produção, disseminação e uso da informação, desde a concepção da ideia até a aceitação dos resultados para compor o estoque internacional de conhecimento (TARGINO, 2000).

Os estudos a respeito da comunicação científica em Moçambique podem ser ainda considerados incipientes, porém, as várias iniciativas tomadas pelas IESP, por exemplo, a realização periódica de eventos científicos, incluindo os programas de pós-graduação, ainda não deram resultados significativos, isto é, ainda não repercutiram na produção científica nacional.

\footnotetext{
${ }^{6}$ GARVEY, W. D. Communication: the essence of science facilitating information among librarians, scientists, engineers and students. Oxford: Pergamon Press, 1979.

InCID: R. Ci. Inf. e Doc., Ribeirão Preto, v. 4, n. 2, Ed. esp., p. 165-184, jul./dez. 2013.
} 
O periódico científico é considerado o principal canal formal de disseminação da ciência e, também, como o canal de comunicação mais utilizado por professores e pesquisadores. Os periódicos científicos são definidos por Targino (1999, p. 68) como "um canal de comunicação formal dos resultados de estudos e pesquisas em cada área do conhecimento, tendo como principal público os cientistas, e que dispõe de mecanismos de controle e aferição de qualidade das informações veiculadas". Desde o seu surgimento no século XVII, os periódicos científicos evoluíram muito e atualmente alcançaram, junto a comunicação científica, nível de prestígio que pode ser comparado àquele atribuído ao livro científico nos séculos XVIII e XIX. O primeiro periódico científico foi o Journal des Sçavants. Este periódico divulgava catálogos de livros, a censura de universidades e informava aos leitores sobre acontecimentos de curiosidade humana. O segundo periódico surgiu um ano depois do primeiro, em 1665, com o título de Philosophical Transactions, da Royal Society of London, da Inglaterra. Com esse novo meio de comunicação científica, os cientistas puderam melhorar as trocas de informações que antes eram feitas por Cartas entre seus pares. Já no Brasil, os primeiros periódicos que surgiram foram a Gazeta Médica do Rio de Janeiro em 1862 e Gazeta Médica da Bahia em 1866.

Hoje, cada vez mais periódicos científicos são criados, e sua disponibilização online facilita o acesso aos mesmos, aumentando potencialmente seu público. A análise da evolução dos periódicos científicos torna evidente a sua importância no processo de comunicação científica. Os periódicos científicos podem ser considerados como o principal meio de divulgação dos resultados de pesquisas e experiências, fato esse que favorece a evolução da própria ciência. Por isso, pode-se dizer que a história dos periódicos científicos se confunde com a evolução da ciência e da pesquisa científica (MEADOWS, 1999).

Com o surgimento das Tecnologias de Informação e Comunicação (TICs), os periódicos científicos sofreram mudanças em sua apresentação e passam a ser oferecidos em vários suportes diferentes, facilitando o acesso à informação e influenciando na forma de utilização das informações. Assim, a partir dos anos de 1990, o periódico impresso começa a receber uma série de críticas. Muitos estudiosos consideram que o periódico impresso tornou-se um veículo moroso, burocrático, limitado tecnicamente e incapaz de dar vazão à crescente produção de artigos nas diversas áreas da pesquisa (CUNHA, 1997, p. 79). Sem negar sua valiosa contribuição 
à história da ciência, estes críticos apontam sintomas de estagnação e esgotamento da indústria de periódicos impressos. Entretanto, Van Brakel$^{7}$ (1995 apud CUNHA, 1997, p. 79) acredita que,

a crise se instaurou porque a publicação científica não se mostra mais capaz de exercer sua função primeira [...] desde sua concepção, a mais importante razão para a existência do periódico científico é a comunicação rápida e ampla dos resultados de pesquisas. Porém, uma série de barreiras vêm fazendo com que o periódico impresso atual se torne menos eficiente como veículo para a divulgação rápida destes resultados [...] as únicas funções em que ele ainda estaria se mostrando eficaz seriam aquelas relativas ao arquivamento, estabelecimento de prioridade autoral e controle de qualidade.

As principais barreiras apontadas na época sobre periódicos impressos, segundo Cunha (1997, p. 79/81), foram: ineficiência; alta especialização e baixa circulação; altos custos; limite físico; esgotamento da indústria (ligado ao problema anterior); falta de espaço nas bibliotecas; falta de agilidade no feedback; sistema de validação. Além de enfrentar todas as críticas apontadas acima, o periódico impresso ainda enfrenta o encantamento provocado pela publicação eletrônica, que já permite a inclusão de recursos variados como som, vídeo, animações, imagens em terceira dimensão, além da interligação hipertextual (no caso específico da Internet) com outras publicações (CUNHA, 1997, p. 81).

Para Meadows (2000), “o periódico eletrônico irá dominar o periódico impresso de maneira muito parecida em relação ao ocorrido com os documentos impressos, que tomaram lugar dos manuscritos”. Segundo Mueller (2006), o periódico científico eletrônico surgiu e ganhou formas inovadoras a partir da década de 90 e despertou nos professores e pesquisadores a esperança de uma mudança radical no sistema tradicional de comunicação científica. Assim, quando surgiu o periódico eletrônico se anunciava como solução para os problemas da comunicação científica, rompiam-se os grilhões que amarravam o artigo científico contemporâneo a uma metodologia e tecnologia ultrapassadas, que estava aquém das necessidades de um mundo cada vez mais globalizado.

De acordo com Briquet de Lemos (2005, p. 7-8),

o periódico eletrônico seria também a libertação dos sistemas perversos impostos pelas editoras de periódicos científicos, inclusive as sociedades científicas, isoladamente, ou a elas associadas. Isso no mundo desenvolvido. No mundo subdesenvolvido a proposta seduzia principalmente pelo seu aparente baixo custo de produção. Era uma proposta que tinha tudo para dar certo. Finalmente a produção científica dos países da periferia

\footnotetext{
${ }^{7}$ VAN BRACKEL, P. A. Electronic journal: publishing via Internet's WWW. The Electronic Library, v. 13, n. 4, p. 389-395, Aug. 1995.
}

InCID: R. Ci. Inf. e Doc., Ribeirão Preto, v. 4, n. 2, Ed. esp., p. 165-184, jul./dez. 2013. 
poderia valer-se de um veículo de divulgação universal, principalmente agora que essa produção vinha redigida numa língua planetária.

As várias denominações existentes para periódicos eletrônicos dificultam uma definição precisa para esse termo. Com base nas definições apresentadas na literatura, entende-se por periódico eletrônico, aquela publicação que pretende ser continuada indefinidamente, que apresente procedimentos de controle de qualidade dos trabalhos publicados aceitos internacionalmente, e que disponibilize o texto completo do artigo através de acesso online, podendo ter ou não uma versão impressa ou em outro tipo de suporte (OLIVEIRA, 2008, p. 71). Portanto, a coexistência dos formatos (impresso e eletrônico) pode ser encarada como um ponto positivo para a aceitação e maior disseminação do suporte eletrônico, pois permite aos professores e pesquisadores experimentarem a versão eletrônica sem, no entanto, abrir mão do prestígio do meio impresso (MEADOWS, 2001).

O senso comum define Portal como local único em que se centralizam e se disponibilizam de modo orgânico todas as informações sobre determinado assunto podem ser encontradas. No entanto, podemos definir os Portais de Periódicos, baseando-se na interação via web, relacionando usuários, sistema e informação, configurando-se, conforme Dias (2001); Costa (2008), em um ponto de interface usado pelos seus usuários na busca, no compartilhamento, na disseminação de informação e na provisão de serviços para os Portais e suas comunidades. Na sua maioria, os Portais de Periódicos estão vinculados às instituições promotoras do conhecimento científico, como instituições de ensino superior e de pesquisa; ordens, conselhos e associações profissionais; associações de pós-graduação; sociedades para o progresso da ciência; dentre outras.

\section{Procedimentos Metodológicos}

A escolha de Instituições de Ensino Superior e de Pesquisa (IESP) moçambicanas justifica-se pelo fato de: (a) na sua maioria, são instituições de grande porte e de referência a nível nacional e internacional; (b) são as instituições que mais utilizam os Portais de Periódicos no desenvolvimento das suas atividades de ensino e pesquisa. E a escolha de periódicos eletrônicos e de Portais de Periódicos de INASP, HINARI, AGORA e CAPES justifica-se pelos seguintes aspectos: (a) são Portais de Periódicos que abrangem todas as áreas do conhecimento;

InCID: R. Ci. Inf. e Doc., Ribeirão Preto, v. 4, n. 2, Ed. esp., p. 165-184, jul./dez. 2013. 
(b) estão situados em ambiente eletrônico e; (c) carência de estudos sobre seu uso em Moçambique.

O objetivo deste estudo permitiu uma opção metodológica que privilegiou os aspectos qualitativos. A escolha da abordagem qualitativa vinculou-se a necessidade de se apresentar estratégias que possibilitassem o incremento do nível de conhecimento e de satisfação das necessidades dos usuários (professores e pesquisadores) em relação aos serviços disponibilizados pelos Portais de Periódicos INASP, HINARI, AGORA e CAPES. De natureza exploratóriadescritiva no presente estudo, procurou-se descrever o perfil do grupo-alvo em relação a sexo, faixa etária, nível de escolaridade. Finalmente, recorreu-se da análise documental através da apreciação de documentos oficiais (decretos, leis, etc.) e técnicos (relatórios, documentos normativos, programas institucionais e governamentais, etc.) coletados junto aos órgãos do Governo de Moçambique, voltados para políticas públicas de acesso à informação.

A amostra desta pesquisa caracterizou-se por ser não probabilística por conveniência e foi composta por professores e pesquisadores oriundos de 20 IESP (o universo do presente estudo) em Moçambique que estão registradas para o acesso aos Portais de Periódicos INASP, HINARI, AGORA e CAPES. Dados não sistematizados indicam que a comunidade de usuários potenciais dos Portais de Periódicos em Moçambique é estimada em, cerca de 3.700 membros, entre professores e pesquisadores. Desta forma, julgamos que era necessário, em função do tempo de execução e dos objetivos pretendidos, selecionar os professores e pesquisadores de IESP que utilizassem efetivamente os Portais de Periódicos. Os professores e pesquisadores que não fazem uso desses recursos eletrônicos foram excluídos da amostra, apesar de estarem inclusos nas IESP inscritas nos Portais, principalmente o Portal do INASP, considerado por nós como foco central da análise.

Com perguntas abertas e fechadas, o questionário foi o principal instrumento de coleta de dados utilizado. Foram distribuídos 155 questionários, dos quais 107 (69\%) foram respondidos por professores e pesquisadores. Também foram aplicadas cinco entrevistas semiestruturada aos bibliotecários de referência que lidam diretamente com a gestão dos Portais de Periódicos de INASP, HINARI, AGORA e CAPES e responsáveis pelo treinamento dos usuários em nível nacional, pela divulgação, reclamações e demandas dos serviços associados aos Portais; utilizando-se como roteiro um questionário específico para cada grupo. 
Aleatoriamente, sem usar nenhuma fórmula estatística ou matemática, a população estudada foi extraída das seis principais instituições de ensino superior (Universidade Eduardo Mondlane, Universidade Pedagógica, Universidade Lúrio, Instituto Superior de Relações Internacionais, Universidade Politécnica de Moçambique, Universidade São Tomás de Moçambique) e de quatro instituições de pesquisa (Centro de Formação Jurídica e Judiciária, Instituto de Investigação Agrária de Moçambique, Instituto de Investigação Pesqueira e Instituto de Coração). Foram entrevistados bibliotecários de referência da Biblioteca Central Brazão Mazula (2) e da Faculdade de Medicina (1), ambas da Universidade Eduardo Mondlane; do Instituto de Investigação Pesquisa (1) e; da Universidade Lúrio (1).

\section{Apresentação e Análise dos Resultados}

Os dados foram agrupados por semelhança de conteúdo em quatro categorias: perfil do usuário; interação com meio eletrônico; uso de periódicos eletrônicos; uso dos Portais de Periódicos de INASP, HINARI, AGORA e CAPES.

Através do perfil sócio-demográfico dos respondentes objetivou-se sistematizar informações sobre a população estudada, tendo em conta as seguintes variáveis: identificação da instituição e da área de conhecimento a qual o respondente pertence, sexo, categoria profissional. Os resultados obtidos demonstram que a Universidade Eduardo Mondlane (UEM) apresenta o maior número de respondentes - 32 (29.9\%) - comparativamente às outras instituições. Esta tendência decorre do fato de a UEM ser a maior e mais antiga Universidade de Moçambique e, durante muito tempo, a única no país. Possui o maior número de professores e oferece 71 cursos no nível de graduação e 32 cursos em nível de pós-graduação (incluindo dois cursos no nível de doutoramento), totalizando 103 cursos. Até o ano de 2010, a UEM contava com 16 unidades acadêmicas, sendo 11 faculdades e cinco escolas (UEM, 2010). Apesar do retorno positivo que tivemos, não houve nenhum respondente da área de Artes, por ser uma área de pesquisa relativamente nova no país. Só em 2008 a Universidade Eduardo Mondlane criou os primeiros cursos de Licenciatura em Música e Teatro. No que diz respeito ao grau de categoria profissional da população amostrada, constatamos que os maiores valores percentuais se encontram ao nível do Assistente Estagiário (31.8\%) e Assistente de Pesquisa (10.3\%), o que de certa forma é 
compreensível se cruzarmos estes valores com os dados relativos à faixa etária, no qual podemos concluir que o universo de usuários de recursos eletrônicos em Moçambique é de 31 a 40 anos.

A interação com meio eletrônico objetivou: (1) identificar as ações governamentais na implementação das políticas públicas de acesso e uso de tecnologias de informação e comunicação (TICs) em Moçambique e; (2) identificar as diferentes etapas de implementação destas políticas pelas IESP, no que se refere à infraestrutura tecnológica, formação e treinamento dos professores e pesquisadores para uso dessas ferramentas. Vale ressalvar que, a pesquisa acadêmica, por envolver diretamente o processo de criação e transmissão do conhecimento, apresenta-se como uma das atividades que mais tem se beneficiado com o uso das TICs, ou seja, a Internet, pois vem proporcionando ao pesquisador acadêmico a facilidade de localizar e recuperar informações geradas por outros pesquisadores através da grande rede (BORBA; COSTA; MARTINS, 2007, p. 82). Para acelerar o desenvolvimento das TICs em Moçambique, o Governo criou a Política de Informática (2000), a Estratégia de Implementação da Política de Informática (2002), a Política de Ciência e Tecnologia (2003), a Estratégia do Governo Eletrônico (2005) e o Projeto MORENET (2008). Apesar do esforço do Governo em estabelecer estas iniciativas, a maior parte das metas projetadas ainda estão em fase de implementação.

Relativamente à frequência de utilização das tecnologias, nota-se um percentual muito levado de uso diário das TICs, o que de certa forma é compreensível se cruzarmos estes valores com os dados relativos ao local de acesso, no qual podemos concluir que se realiza nas salas de trabalho dos respondentes e nas bibliotecas das instituições onde eles realizam as suas atividades de ensino e pesquisa. Os resultados obtidos sobre o tempo dedicado às atividades de pesquisa, $o$ maior índice recai sobre os usuários que se dedicam entre 6 a 10 horas à pesquisa. Este fator se justifica pela ausência de financiamentos para a pesquisa, a inexistência de infraestruturas tecnológicas básicas, fraca conectividade e comunicação de dados. Podemos, então concluir que, em relação a interação com o meio eletrônico, as respostas foram muito variadas. Entretanto, salientamos que, todos os usuários utilizam as TICs, principalmente a Internet e não foi encontrada relação entre o número de recursos das TICs utilizado e o tempo gasto para pesquisa, ou seja, de acordo com as respostas, não se pode afirmar que quanto maior o número de recursos eletrônicos usados, maior o tempo que o usuário dispende no desenvolvimento da pesquisa.

InCID: R. Ci. Inf. e Doc., Ribeirão Preto, v. 4, n. 2, Ed. esp., p. 165-184, jul./dez. 2013. 
Em relação ao uso de periódicos eletrônicos, objetivou-se analisar a utilização dos periódicos eletrônicos nas IESP em Moçambique, com finalidade de compreender o nível de conhecimento que os professores e pesquisadores têm relativamente à existência destes recursos. Os resultados obtidos apontam que, cerca de $72 \%$ dos respondentes utilizam os periódicos eletrônicos nas suas atividades de ensino e pesquisa. Relativamente às razões que levam $28 \%$ a não utilizar estes periódicos, concluímos que a grande maioria ainda desconhece estes recursos. Observa-se, sobretudo, que são os professores que mais usam os periódicos eletrônicos (72.1\%), em oposição àqueles que menos os usam, pesquisadores (27.9\%). Talvez pelo fato de, os professores estarem naturalmente melhor informados quanto à existência, utilização e acesso a estes periódicos, não só, as instituições de ensino superior foram pioneiras a aderirem à iniciativa de acesso aberto aos recursos eletrônicos no país. Os resultados da observação apontam para a confirmação da hipótese de que os usuários fazem pouco uso dos periódicos eletrônicos, apesar dos dados obtidos indicarem o lado oposto da nossa hipótese. Na prática verifica-se que a maioria utiliza frequentemente os acervos bibliográficos disponíveis nas bibliotecas.

Relativamente à idade, constatou-se que os pesquisadores mais velhos apresentam menor preferência pelo formato eletrônico. Não obstante, são várias razões indicadas para não utilização dos periódicos eletrônicos, entre elas, questões de natureza tecnológica e de desconfiança por parte das gerações mais velhas.

O estudo acerca do uso dos Portais de periódicos objetivou contribuir para a otimização do uso dos Portais de INASP, HINARI, AGORA e CAPES, na medida em que se buscou conhecer o grau de satisfação dos usuários em relação aos Portais e perceber a sua influência no processo de ensino e pesquisa científica em Moçambique. O Portal de INASP ${ }^{8}$ foi criado, em 1992, pelo Conselho Internacional para a Ciência (International Council for Science), visa melhorar o acesso à informação e ao conhecimento por meio de um compromisso para a construção de capacidades nos países emergentes e em desenvolvimento. Sua missão é melhorar o fluxo de informação científica e acadêmica entre os países, especialmente aqueles com sistemas menos desenvolvidos de publicação e divulgação científica. O principal papel do INASP é negociar com as editoras internacionais, em nome dos países parceiros, para adquirir a baixo

\footnotetext{
${ }^{8}$ Disponível em: http://www.inasp.info/file/3d034b8bae0a3f7e1381979aedc356a9/about-inasp.html. Acessado em: 23 jan. 2013.
}

InCID: R. Ci. Inf. e Doc., Ribeirão Preto, v. 4, n. 2, Ed. esp., p. 165-184, jul./dez. 2013. 
custo os periódicos eletrônicos e e-books. O acesso aos periódicos eletrônicos disponíveis no Portal é através do IP (Internet Protocol).

O Portal HINARI $I^{9}$ foi lançado oficialmente em 2002. É coordenado pela Organização Mundial da Saúde (OMS) (em inglês, World Health Organization - WHO), com o apoio da Biblioteca da Universidade de Yale. Tem por principal objetivo disponibilizar informações em ciências da saúde para instituições governamentais acadêmicas e de pesquisa que desenvolvem políticas de saúde nos países em desenvolvimento. O Programa HINARI trabalha em parceria com os Programas AGORA e OARE. O Programa AGORA ${ }^{10}$ (Access to Global Online Research in Agriculture), que é gerido pela FAO (Organização das Nações Unidas para a Agricultura e Alimentação) permite o acesso eletrônico à pesquisa global em agricultura. Enquanto, o Programa OARE (Online Access to Research in the Environment) permite o acesso eletrônico à pesquisa sobre o meio ambiente e é liderado pela UNEP (Organização das Nações Unidas para o Meio Ambiente), a Universidade de Yale e o Programa ARDI (The Access to Research for Development and Innovation) e é coordenado pela Organização Mundial da Propriedade Intelectual (WIPO). O acesso as bases de dados desses Portais é através de senha.

O Portal de Periódicos de Capes $^{11}$ é uma biblioteca virtual que reúne e disponibiliza às instituições de ensino e pesquisa no Brasil o melhor da produção científica internacional. Foi criado tendo em vista o deficit de acesso das bibliotecas brasileiras à informação científica internacional, na perspectiva de que seria demasiadamente caro atualizar esse acervo com a compra de periódicos impressos para cada uma das universidades do sistema superior de ensino federal. Foi desenvolvido ainda com o objetivo de reduzir os desnivelamentos regionais no acesso a essa informação no Brasil. Ele é considerado um modelo de consórcio de bibliotecas único no mundo, pois é inteiramente financiado pelo Governo brasileiro. Atualmente, o Portal reúne e disponibiliza a instituições do ensino e pesquisa no Brasil, a produção científica nacional e internacional. Possuem acesso livre e gratuito ao conteúdo do Portal de Periódicos alunos, professores, pesquisadores e funcionários vinculados às instituições.

O acesso e uso dos Portais de Periódicos de INASP, HINARI, AGORA e CAPES pelos alunos, professores e pesquisadores das instituições de ensino superior e de pesquisa em

\footnotetext{
${ }^{9}$ Disponível em: http://www.who.int/hinari/en/. Acessado em: 24 jan. 2013.

${ }^{10}$ Disponível em: http://www.aginternetwork.org/en/. Acessado em: 24 jan. 2013.

${ }^{11}$ Disponível em: http://www.periodicos.capes.gov.br/. Acessado em: 24 jan. 2013.
} 
Moçambique, é intermediado pela Direção dos Serviços de Documentação, da Universidade Eduardo Mondlane. Os periódicos nacionais ainda não estão disponíveis nos Portais, porque, os poucos que existem, são editados e publicados em formato impresso. O uso dos Portais é gratuito para os usuários autorizados das instituições de ensino superior e de pesquisa registradas nos Portais.

Os dados obtidos evidenciam que 72.9\% dos usuários acessavam os Portais, contra 27.1\% que nunca usou os Portais. Foram apontados como dificuldades e barreiras para o uso, a lentidão do acesso ao sistema e as dificuldades de acesso fora de instituições registradas nos Portais. No entanto, podemos salientar que, o alto índice de usuários (professores e pesquisadores) que desconhecem os Portais, apesar de menor preocupa tendo em conta os investimentos gastos nas assinaturas junto as editoras de periódicos, pois demonstra a necessidade de maior divulgação dos Portais nas instituições de ensino superior e de pesquisa no país. Verificam-se dificuldades de acesso ao texto integral relacionado quer com problemas de ordem técnica quer com pedidos de senhas e IP de acesso, acesso aos números antigos, entre outros, foram apontados como as grandes desvantagens ao uso dos periódicos eletrônicos.

As barreiras tecnológicas por que passam as IESP, tais como: problemas na utilização e configuração da rede; tempo de expiração de determinados recursos; baixa velocidade de resposta; problemas de acesso a Internet e as bases de dados; dificuldades em acessar os Portais fora do ambiente da instituição; problemas técnicos ou operacionais de conexão ou da rede e; pouca disponibilidade de salas ou laboratórios com Internet. As barreiras idiomáticas foram apontadas como maior dificuldade para da realização das pesquisas de acordo com os respondentes, o que se compreender pelo fato de a língua oficial em Moçambique ser a língua portuguesa. Aliás, Moçambique é um país multilingue e multicultural. Todavia, imperativos de ordem política e também de ordem técnica, quando da Independência, ditaram que a língua de ensino e de pesquisa em todo o sistema nacional de educação e do sistema nacional de pesquisa fosse o português, a língua oficial. Portanto, pode-se afirmar sem dúvida, que o acesso e uso dos Portais de Periódicos, até então verificado, devem-se ao esforço da Direção dos Serviços de Documentação, da Universidade Eduardo Mondlane em divulgar estes recursos entre as instituições de ensino superior e de pesquisa em Moçambique.

InCID: R. Ci. Inf. e Doc., Ribeirão Preto, v. 4, n. 2, Ed. esp., p. 165-184, jul./dez. 2013. 


\section{Considerações Finais}

Apesar dos avanços alcançados pelo setor de educação, ciência e tecnologia, o subsistema do ensino superior é caracterizado pela existência de muitos cursos em áreas de Ciências Humanas e Sociais, pela escassez de cursos de pós-graduação e pela existência de um grande número de professores na categoria de "Assistente Estagiário", fatores que têm influenciado a produção científica. Constatamos também que, o sistema nacional de pesquisa enfrenta os mesmos problemas, com maior número de pesquisadores na categoria de "Assistente de Pesquisa". Ademais, as várias iniciativas governamentais implantadas, algumas ainda em fase de implementação, deram os primeiros passos no sentido de oportunizar maior acesso à informação, mas necessita ainda de um enfoque mais estratégico e investimentos para garantir que todos os cidadãos possam aceder às tecnologias.

Os resultados obtidos permitem considerar que tanto os professores, quanto os pesquisadores de instituições de ensino superior e de pesquisa em Moçambique utilizam de forma rotineira periódicos eletrônicos em suas atividades de ensino e pesquisa. No entanto, a cultura impressa ainda está fortemente enraizada. Todavia, podemos concluir que a tendência é para o aumento da circulação e utilização do formato eletrônico em detrimento do impresso. Contudo, ainda há muitos profissionais, sobretudo, nas categorias de "Professor Catedrático", "Professor Associado" e "Pesquisador Principal", que apesar da experiência acadêmica e tempo de exercício da profissão, desconhecem a existência dos periódicos eletrônicos e dos Portais de Periódicos.

Os profissionais que dizem conhecer e utilizar os recursos eletrônicos em Moçambique opinam que é necessário maior divulgação dos Portais de Periódicos e a realização de mais cursos de treinamento para alunos, professores e pesquisadores. Quanto ao grau de satisfação em relação ao uso dos Portais, os mesmos estão satisfeitos, apesar de apontar o idioma como um grande entrave. Do ponto de vista dos profissionais da informação, embora os bibliotecários de referência de IESP reconheçam as vantagens e a importância dos recursos eletrônicos, constatamos que questões como idioma, bloqueio de acesso a alguns artigos, cancelamento de periódicos, pagamento tardio das assinaturas, acesso permanente e a transferência de títulos entre editores constituem as principais preocupações.

InCID: R. Ci. Inf. e Doc., Ribeirão Preto, v. 4, n. 2, Ed. esp., p. 165-184, jul./dez. 2013. 
Em Moçambique, os recursos eletrônicos, em particular os periódicos eletrônicos e Portais de Periódicos, ainda têm muito a serem explorados. Acredita-se que a pesquisa cumpriu seu objetivo no que se refere ao mapeamento proporcionado, possibilitando a identificação de novas demandas de estudo com base nos dados sistematizados. Espera-se que os resultados sirvam, não só de indicadores, como também de suporte para a avaliação das fontes de informação e dos serviços oferecidos pelos Portais de Periódicos em Moçambique. Para finalizar, concluímos que, serão necessários novos estudos baseados em metodologias de acesso e uso dos Portais de Periódicos centradas no usuário, estudos específicos para cada IESP com o objetivo de compreender certos aspectos sobre as necessidades de informação, demandas e usos da informação pelos usuários dos Portais de Periódicos INASP, HINARI, AGORA e CAPES.

Assim, recomenda-se a realização de estudos verticais para cada instituição de ensino superior e/ou de pesquisa e para cada grupo de usuários (alunos, professores ou pesquisadores). Devido à grande complexidade percebida, indica-se que a necessidade de realização dos estudos específicos dedicados a cada Portal, particularmente para os Portais de INASP e CAPES. Para tanto, recomenda-se estudos qualitativos com os usuários. Recomenda-se, também, a realização de estudos por área de conhecimento ou agrupando-as por semelhança e aproximação para identificar as áreas e subáreas que ainda não estão sendo atendidas pelos atuais Portais, bem como as condições do atendimento já realizado. Recomenda-se a realização de estudos sobre a capacitação de recursos humanos. Para tanto, pode se utilizar o sistema nacional de educação como fator-chave da inclusão digital das novas gerações através da formação de professores, equipando as escolas e adaptando os curricula. Formar um novo perfil do profissional técnico polivalente com foco nas TICs. Investir na capacitação massiva para a utilização das tecnologias digitais, incluindo no uso de software livre.

InCID: R. Ci. Inf. e Doc., Ribeirão Preto, v. 4, n. 2, Ed. esp., p. 165-184, jul./dez. 2013. 


\section{Referências}

ARAÚJO, E. A. et al. Periódico eletrônico Informação \& Sociedade: Estudos - impactos no contexto da comunicação científica. Perspectivas em Ciência da Informação. Belo Horizonte, v. 11, n. 3, p. 335-347, set./dez. 2006.

BORBA, M. S. A.; COSTA, G. C. N.; MARTINS, R. A. C. O periódico científico online e sua importância para a pesquisa. Interface, Natal, v. 4, n. 2, p. 79-94, jul./dez. 2007.

BRIQUET DE LEMOS, A. A. Publicar e perecer. Ciência da Informação, Brasília, v. 34, n. 2, p. 7-8, maio/ago. 2005. Disponível em:

http://www.ibict.br/cienciadainformacao/viewissue.php?id=9. Acesso em: 5 set. 2011.

CASTEDO, R. S. Revistas científicas on-line de comunicação no Brasil: a produção editorial sob o impacto da tecnologia digital - Volume I. 2009. Dissertação (Mestrado em Comunicação e Informação) - FABICO/UFRGS, 2009.

CENDÓN, B. V. et al. Uso dos periódicos do Portal CAPES pelos Programas de Pós-graduação da Faculdade de Odontologia de Araraquara UNESP no período de 2000 a 2005.

Transinformação. Campinas, v. 21, n. 2, 133-149, maio/ago. 2009.

CENDÓN, B. V.; RIBEIRO, N. A. Análise da literatura acadêmica sobre o Portal Periódicos Capes. Informação \& Sociedade, João Pessoa, v. 18, p. 157-178, 2008.

COSTA, M. T. F. O uso de periódicos científicos electrónicos nas instituições do ensino superior público em Portugal. 2008. Dissertação (Mestrado em Ciências da Documentação e Informação) - FL/Universidade de Lisboa, 2008.

CRUZ, A. A. A. C. et al. Impacto dos periódicos eletrônicos em bibliotecas universitárias. Ciência da Informação, v. 32, n. 2, p.47-53, 2003.

CUNHA, A. A. L.; CENDÓN, B. V. Uso de bibliotecas digitais de periódicos: um estudo comparativo do uso do Portal de Periódicos CAPES entre as áreas do conhecimento. Pespectivas em Ciência da Informação, v. 15, n. 1, p. 70-91, jan./abr. 2010.

CUNHA, L. Publicações científicas por meio eletrônico: critérios, cuidados, vantagens e desvantagens. Perspectivas em Ciência da Informação, Belo Horizonte, v. 2, n. 1, p. 77-92, jan./jun. 1997.

DIAS, C. A. Portal corporativo: conceitos e características. Revista Ciência da Informação, v. 30, n. 1, p.50-60, jan./abr. 2001.

DIAS, G. A. Periódicos científicos eletrônicos brasileiros na área da ciência da informação: análise das dinâmicas de acesso e uso. 2009. Tese (Doutorado) - ECA/USP, 2009. 
LANCASTER, F. W. The evolution of electronic publishing. Library Trends, v. 43, n. 4, p. 518-527, 1995.

MAIA, L. C. G. Uso de periódicos eletrônicos: um estudo sobre o Portal de Periódicos CAPES na UFMG. 2005. Dissertação (Mestrado em Ciência da Informação) - ECI/UFMG, 2005.

MANGUE, M. V. Consolidação do processo de informatização em sistemas de bibliotecas universitárias da África do Sul, Brasil e Moçambique. 2007. Tese (Doutorado em Ciência da Informação) - ECI/UFMG, 2007.

MEADOWS, A. J. A comunicação científica. Brasília: Briquet de Lemos, 1999.

Avaliando o desenvolvimento da comunicação científica. In: MULLER, S. P. M.;

PASSOS, E. J. L. (Org.). Comunicação científica. Brasília: UnB, 2000. p. 23-34.

MEADOWS, A. J. Os periódicos científicos e a transição do meio impresso para o eletrônico.

Revista de Biblioteconomia de Brasília, Brasília, v. 25, n. 1, p. 5-14, jan./jun. 2001.

MOÇAMBIQUE. Lei n. 1/93, de 24 de Junho. Regula a actividade do ensino superior na República de Moçambique. Boletim da República, n. 25, p. 122(1)-122(6).

MONTEIRO, M. H. W. Ciência e tecnologia: produção de conhecimento em Moçambique entre 2003 a 2008. 2010. Dissertação (Mestrado em Sociologia) - IFCH/UFRGS, 2010.

MUELLER, S. P. M. A comunicação científica e o movimento de acesso livre ao conhecimento. Ciência da Informação, Brasília, v. 35, n. 2, p. 27-38, maio/ago. 2006.

NHARRELUGA, R. S. O governo eletrônico em Moçambique: uma reflexão sobre políticas públicas de informação. 2006. Dissertação (Mestrado) - UFF/IBICT, 2006.

OLIVEIRA, E. B. P. M. Periódicos científicos eletrônicos: definições e histórico. Informação \& Sociedade: Estudos, João Pessoa, v. 18, n. 2, p. 69-77, maio/ago. 2008.

Uso de periódicos científicos por docentes e pós-graduandos no Instituto de

Geociências da USP. 2006. Dissertação (Mestrado) - ECA/USP, 2006.

PEEK, R. P.; POMERANTZ, J. P. Electronic scholarly journal publishing. Annual Review of Information Science and Technology, v. 33, p. 321-356, 1998.

ROWLANDS, I. Electronic journals and user behavior: a review of recent research. Library \& Information Science Research, v. 29, p. 369-396, 2007.

TARGINO, M. G. Comunicação científica na sociedade tecnológica: periódicos eletrônicos em discussão. Comunicação \& Sociedade, São Paulo, n. 31, 1999. 
A região geográfica como fator interveniente na produção de artigos de periódicos científicos. In: MUELLER, S. P. M.; PASSOS, E. J. L. (Org.). Comunicação científica. Brasília: UnB, 2000. p. 51-72.

ZIMBA, H. F. A dimensão política e o processo de institucionalização da ciência e tecnologia em Moçambique. 2010. Tese (Doutorado em Ciência da Informação) - FACECI/UnB, 2010.

DOI: $\underline{10.11606 / \text { issn.2178-2075.v4i2p165-184 }}$

InCID: R. Ci. Inf. e Doc., Ribeirão Preto, v. 4, n. 2, Ed. esp., p. 165-184, jul./dez. 2013. 